\title{
A DISCRETE MODEL OF CRACK GROWTH BY STRESS-ASSISTED MIGRATION/AGGREGATION OF POINT DEFECTS
}

\author{
Athanios G. Konstandopoulos* and Elias C. Aifantis** \\ Division of Mechanics, School of Engineering \\ Aristotle University, Box 42254006 Thessaloniki, Greece.
}

\begin{abstract}
The problem of crack propagation is studied as a cluster growth process with the aid of discrete computer 'experiments'. A mechanistic ballistic aggregation model motivated by the continuum theory of stress-assisted migration of point defects is formulated and simulated on a square lattice. Preliminary results reported here show that crack propagation becomes possible only when a model parameter $r_{0}$ expressing the size of the influence region of the crack tip stress field, increases beyond a critical value $r_{\text {crit }}$. The crack tip velocity is found to follow a power law with respect to $\left(r_{0}-r_{c r i t}\right)$ while the fractal dimension of the generated cracks scales with $r_{0}$.
\end{abstract}

\section{INTRODUCTION}

Among the central open problems in fracture mechanics are that of predicting crack shapes and morphologies, as well as the crack-tip velocity of propagation $v_{c}$. One hopes that, eventually the rich variety of crack morphologies seen in experiments and in nature will be connected to the dominant mechanisms of crack growth. In addition, determination of $v_{c}$ should become possible as part of the problem solution, rather than imposing it apriori as is usually done in elastodynamic or pseudoplastic analyses of crack propagation.

Considerable activity has been devoted lately to computer simulations of spring networks (1) with local bond breaking criteria dependent on the prevailing stress (and/or its gradient) level, in the interest of studying fracture processes in disordered media. The focus of that research has been the study of crack morphologies using fractal geometry (2). Although simple in concept, such simulations pose a great challenge due to the very large super-computing resources required (1). Another line of research, using discrete 'cluster growth' models, has also provided valuable insight into the connection between microscopic growth mechanisms and resulting structure/patterns in a variety of aggregation/deposition phenomena, see e.g. Meakin (3)

\footnotetext{
*Present address: MicroPhenomena, 45 Tselepi Street, 54352 Thessaloniki, Greece.

**Also Center for Mechanics of Materials \& Instabilities, Michigan Tech, Houghton, MI 49931, USA.
} 
for a review. The present study of the problem of crack propagation/morphology, is motivated by this latter approach. Crack propagation is viewed as a growth process occuring by the 'aggregation' of carriers of disorder in the material (e.g. point defects such as vacancies or microvoids and interstitials) at the advancing crack tip. In our mechanistic, discrete model of crack growth these carriers of disorder move accordingly to previously established transport equations. In particular, we consider crack growth due to the stress-assisted migration (4) of defects towards the crack tip. We illustrate the approach using a simple example case i.e. crack growth by microvoid aggregation. We also comment on addressing the case of hydrogen-assisted crack growth.

\section{MODEL FORMULATION}

The starting point of the model is a square $(L \times L)$ lattice (Fig. 1) with a fraction $\omega$ (a model parameter) of its cells considered to be microvoids (from now on to be referred for simplicity as voids), uniformly distributed. A seed cell is placed at $(0$, $L / 2$ ) representing the tip of a crack embedded in the medium. Lattice cells can be in any of three states: void, solid and crack. Void cells that come to within a lattice unit from the crack are assumed to become irreversibly part of the crack. To model in a simple way the activated nature of the process whereby defect motion is expected to start when the local hydrostatic stress level exceeds a critical value, an influence length parameter $r_{0}$ is introduced. Defects at a distance beyond $r_{0}$ from the current position of the crack tip are assumed to stay motionless. In the present context $r_{0}$ is a model control parameter that can be related to the ratio of the applied stress at boundary and the critical stress required to initiate defect motion. The simulation then proceeds as follows:

1. Each time step cells that are within $r_{0}$ are advanced one lattice unit based on the local velocity, which is taken to be proportional to the gradient of the local hydrostatic stress provided by the elastic field. The stress field is assumed to be given by the classical elasticity solutions near crack-tips. For the simulations presented here we have considered Mode I plane stress conditions. It can be then shown that the migration velocities along the $x$ and $y$ axes have the following spatial dependencies:

$$
\begin{aligned}
V_{x} & \sim r^{-3 / 2} \cos \frac{\theta}{2}(1-2 \cos \theta) \\
V_{y} & \sim r^{-3 / 2} \sin \frac{\theta}{2}(1+2 \cos \theta)
\end{aligned}
$$

where $(r, \theta)$ are the polar coordinates from the crack tip.

2. Local transition probabilities for each void for the $x$ and $y$ directions of the lattice are then computed from the following relations: 


$$
\begin{gathered}
p_{x}=(1+\tan \phi)^{-1} \\
p_{y}=\tan \phi(1+\tan \phi)^{-1} \\
\phi=\arctan \left(V_{y} / V_{x}\right)
\end{gathered}
$$

and all voids within $r_{0}$ of the crack tip are advanced one lattice cell in the most probable direction determined with the aid of a random number generator.

3 . Voids that come into contact with cells occupied by the crack are irreversibly marked as 'crack cells' and a new crack tip is defined as the crack cell extending furthest into the lattice in the $x$ direction and in contact with the highest number of neighboring crack cells. In case of a tie, a cell is chosen at random from the set of eligible cells to become the new crack tip.

4. Void velocities are computed with respect to the new crack-tip and the process is repeated from item 1 for a desired number of iterations.

\section{RESULTS AND DISCUSSION}

Examples of cracks generated by the present model are shown in Fig. 2 for different values of $r_{0}$. Notice that as $r_{0}$ becomes comparable to the lattice size finite size effects set in, resulting in a deviation of the crack direction of propagation from the horizontal direction. Characterization of the fractal dimension, $D_{f}$ of the simulated cracks of Fig. 2 indicates that $D_{f}$ increases with $r_{0}$ (Fig. 3a, 3b) During the simulation we also monitor the (discontinuous) crack-tip displacement (Fig. 4), the average slope of which provides an estimate of a continuous crack-tip velocity, $v_{c}$. Fig. 5 depicts the dependence of $v_{c}$ on $r_{0}$ and shows the critical nature of the process whereby crack propagation is possible only for $r_{0}>r_{\text {crit }}$. The power law exponent $\nu$ is very close to 0.5 :

$$
v_{c} \sim\left(r_{0}-r_{c r i t}\right)^{\nu}
$$

For the particular void concentration used $(\omega=0.02) r_{\text {crit }}$ was about 73 . In a subsequent communication (6) we will examine the dependence of $\nu$ and $r_{\text {crit }}$ on $\omega$.

We have deliberatedly neglected Fickean diffusion fluxes in our simulation for simplicity. It can be shown that formally this case corresponds to the limit of large Peclet number, $\mathrm{Pe}$ ( $\equiv M \sigma_{\infty} / D$, a dimensionless number indicating the importance of stress-assisted migration relative to Fickean diffusion). In the previous relation, $M$ is the phenomenological coefficient of stress-assisted diffusion and $D$ the defect Fickean diffusivity. Inclusion of Fickean diffusion (i.e. finite $P e$ ) is expected to 'smear out' the critical nature of the $v_{c}$ growth with respect to $r_{0}$ (Fig. 5).

The case of environmental degradation due to hydrogen (or other corrosive species) diffusion to the crack tip can also be addressed within the present framework. In this case a random walk implementation of the following stress assisted diffusion flux law (4) can be obtained computing 'transition probabilities' of random 


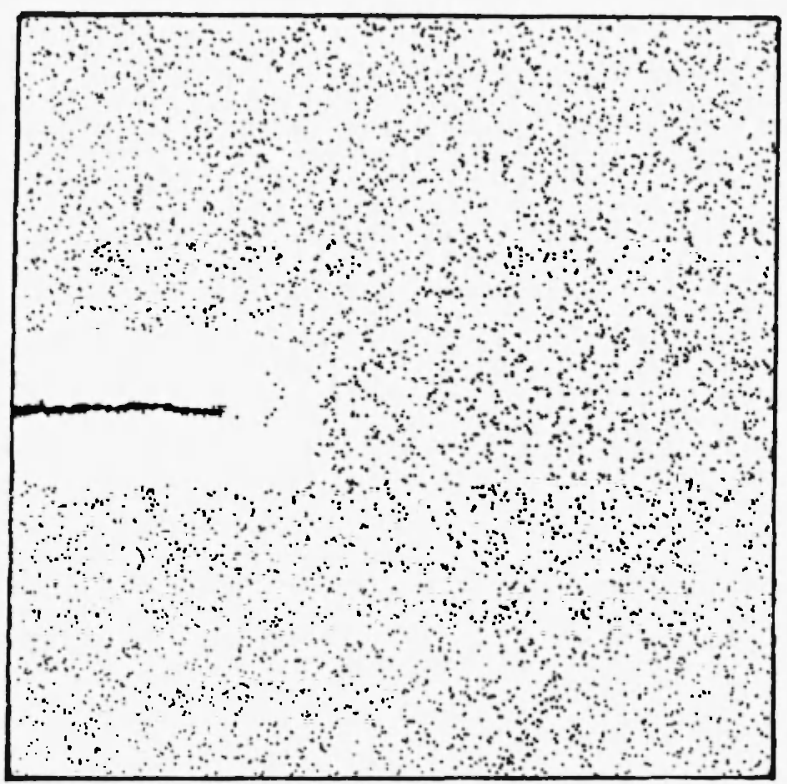

Fig. 1 Schematic of crack growth by void aggregation. Lattice size $\mathrm{L}=512$, void fraction $\omega=0.02$.

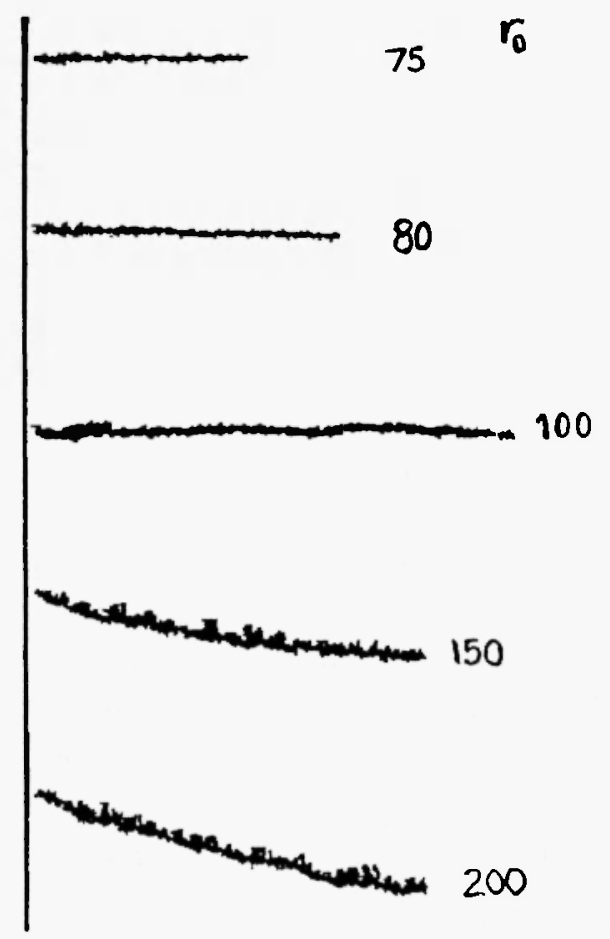

Fig. 2 Crack morphology as a function of $r_{0}$. 

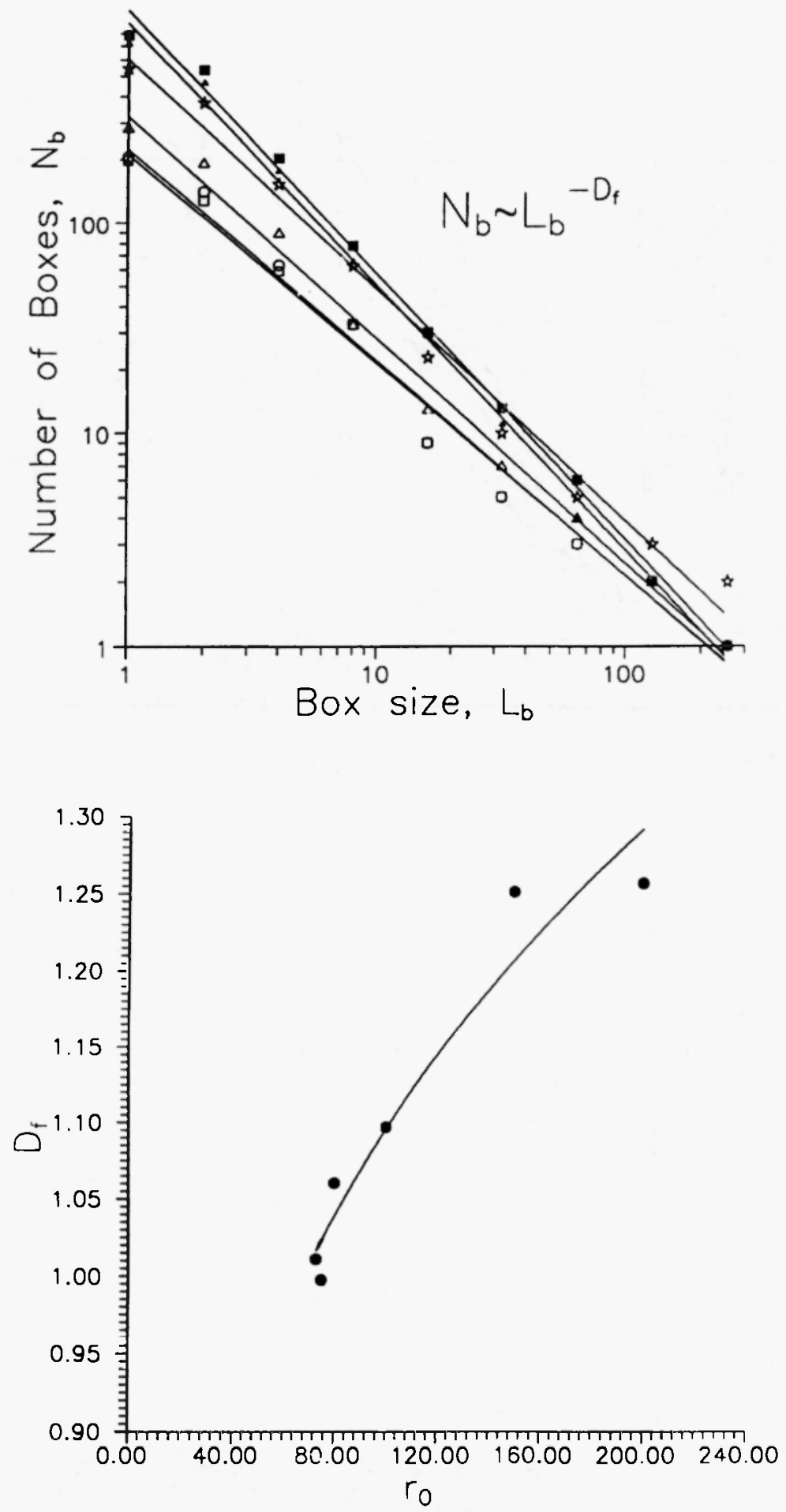

Fig. 3 (a) Determination of crack fractal dimension $D$, by the box-counting method (2). (b) Dependence of $D$, on $r_{0}$. 


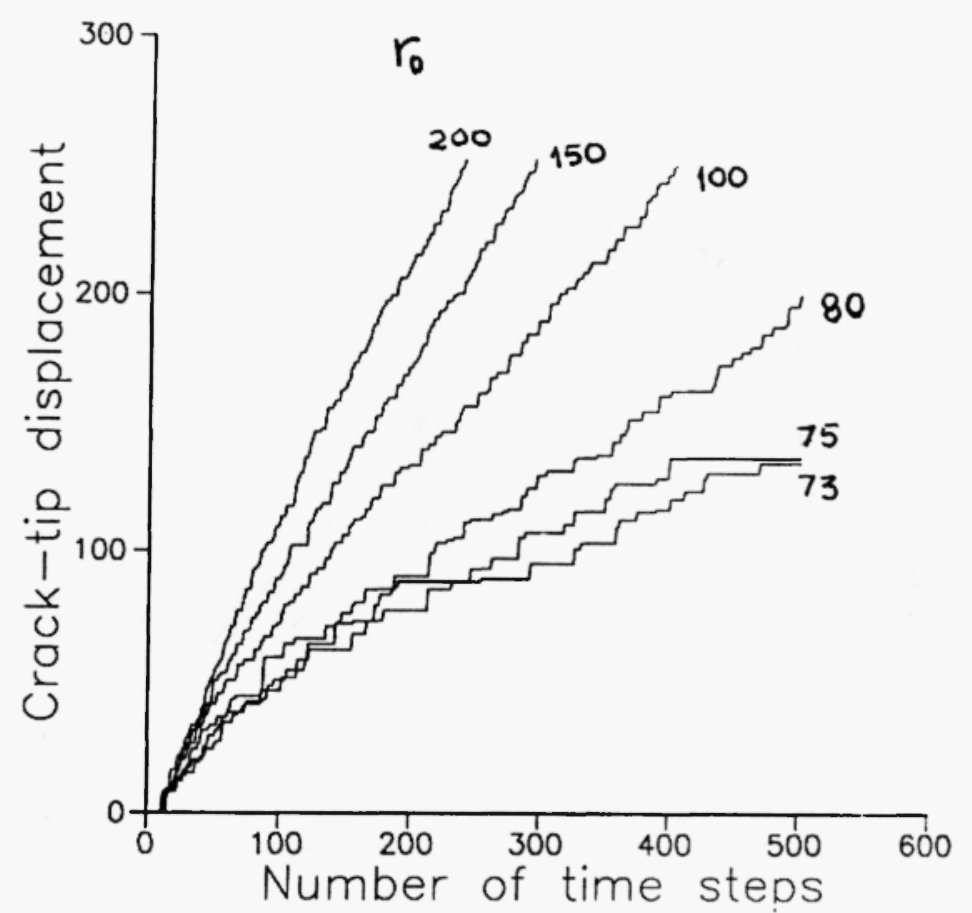

Fig. 4 Evolution of crack tip displacement for various values of $r_{0}$.

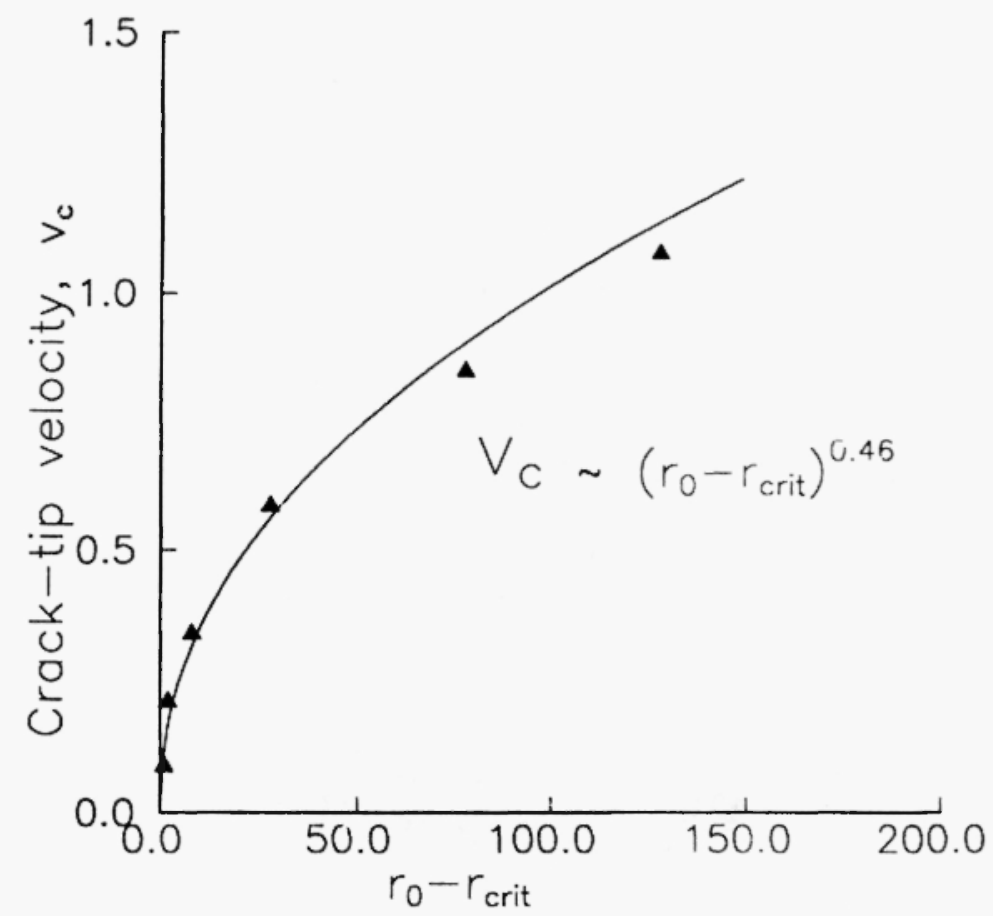

Fig. 5 Crack tip velocity dependence on $\left(r_{0}-r_{\text {crit }}\right)^{\nu}$. For the present simulations $r_{\text {crit }} \approx 73$ and $\nu \approx 0.5$. 
walkers on the lattice cells, along with a hopping probability dependent on the local hydrostatic stress level $\sigma$, in order to model the activated nature of the process:

$$
\mathbf{j}=-D \nabla \rho+M \rho \nabla \sigma
$$

$\mathbf{j}$ is the total species flux while $\rho$ is their concentration. The crack would then grow by 'consuming' regions ahead of the crack tip where the corrosive species concentration exceeds a critical value (see Gdoutos and Aifantis (5) for a continuum mechanics motivation). This case along with extensive parametric studies of the present model will be addressed in a forthcoming paper (6).

While still in preliminary form, it is anticipated that the present approach will provide new insight into crack propagation phenomena and complement recent continuum approaches to crack stability and growth $(7,8)$.

\section{Acknowledgements}

Support of the U.S. National Science Foundation under grant No. INT-8914718 and NATO grant 890480 is gratefully acknowledged. The paper has benefited from a set of incomplete calculations performed by C.C. Cusack under the guidance of ECA. Finally, support from EEC under contract No ER-BCHBGCT 920041 and NSF under grant MSS - 9310476 is also acknowledged.

\section{REFERENCES}

1. Herrmann, H. J. and Roux, S., Statistical Models for the Fracture of Disordered Media, North-Holland, Amsterdam (1990)

2. Mandelbrot, B. B. The Fractal Geometry of Nature, Freeman, New York (1983)

3. Meakin, P. in The Fractal Approach to Heterogeneous Chemistry, Ed. Anvir, D., Wiley, New York, 131 (1989)

4. Aifantis, E. C., Acta Mech., 37, 265 (1980)

5. Gdoutos, E. E. and Aifantis, E. C., Engng. Fracture Mech., 23, 423 (1986)

6. Konstandopoulos, A. G. and Aifantis, E. C., in preparation (1994)

7. Aifantis, E. C. in Constitutive Laws for Engineering Materials - Theory and Applications, Eds. Desai, C. S. et al., ASME Press, New York, 493 (1991)

8. Herrmann, H. J. in Growth Patterns in Physical Sciences and Biology, NATO ASI Series B:304, Eds. Garcia-Ruiz et al., Plenum, New York, 299 (1993) . 